\title{
ON WEIGHTED TIME-OPTIMAL CONTROL FOR LINEAR HYBRID AUTOMATA USING QUANTIFIER ELIMINATION
}

\author{
Yan Pang * Michael P. Spathopoulos* \\ * Department of Mechanical Engineering, University of \\ Strathclyde, Glasgow, UK \\ \{ypang,mps\}@mecheng.strath.ac.uk
}

\begin{abstract}
This paper considers the optimal control problem for linear hybrid automata. In particular, it is shown that the problem can be transformed into a constrained optimization problem whose constraints are a set of inequalities with quantifiers. Quantifier Elimination (QE) techniques are employed in order to derive quantifier free inequalities that are linear. The optimal cost is obtained using linear programming. The optimal switching times and optimal continuous control inputs are computed and used in order to derive the optimal hybrid controller. Our results are applied to an air traffic management example. Copyright (c) 2004 IFAC
\end{abstract}

Keywords: hybrid automata, quantifier elimination, time optimal control

\section{INTRODUCTION}

A mathematical model for hybrid systems is the hybrid automaton, which represents discrete components by finite-state machines and continuous components by real-numbered variables. An important subclass of hybrid systems is the so called rectangular hybrid automata (Preußĩg et al., 1998), where in each mode the continuous dynamics are given as constant differential inclusions. When linear continuous dynamics with both control and $d$ isturbance inputs are considered the so called linear hybrid automata (Xia et al., 2002) are derived.

The optimal control problem, particularly the time-optimal or weighted time-optimal problem is considered in this paper. We derive a hybrid control strategy that drives the system from an initial state into the target set with minimal cost. Assuming that there exists a trajectory starting from the initial state to the target set, the constraints that the trajectory has to satisfy are converted into a set of inequalities with quantifiers. A set of quantifier-free inequalities with the switching times as variables is obtained using quantifier elimination tool QEPCAD (Collins and Hong, 1991). As the quantifier free inequalities are linear, linear programming is used in order to obtain the optimal cost and the associated optimal switching times. Based on the optimal switching times, the optimal continuous control inputs are derived and an optimal controller is constructed.

This paper is organized as follows. In section 2 , we review the basic definitions and concepts related to linear hybrid automata. In section 3 we employ quantifier elimination for the optimal control of rectangular hybrid automata and design the corresponding optimal controller. In section 4 , the optimal control problem for linear hybrid automata is solved. The air traffic management application is illustrated in section 5 . Section 6 contains conclusions.

\section{MODEL AND PROBLEM STATEMENT}

\subsection{Hybrid Automata}

Hybrid automata are generalized finite-state machines where discrete transitions transfer the sys- 
tem between a finite number of modes $Q$. We use $A$ to denote a hybrid automaton. In each discrete mode $q$, the system evolves continuously according to a set of differential equations :

$$
\dot{x}_{q}=f_{q}(x(t), u(t), d(t))
$$

where $x \in X \subseteq \mathbb{R}^{n}, u(t) \in U$ is the continuous control input and $d(t) \in D$ is disturbance input. It is assumed that for every $q \in Q, f_{q}$ is globally lipschitz to ensure the existence and uniqueness of solutions of the differential equation. Furthermore for each mode $q$ there may be a set of state invariant constraints Inv : $Q \rightarrow 2^{X}$ that have to be fulfilled. $\Sigma$ is the set of discrete events and the discrete transition set is $E \subseteq Q \times \Sigma \times Q$. We use guard $G:(Q \times \Sigma \times Q) \rightarrow 2^{X}$ to express the switching conditions that when $x \in G_{q q^{\prime}}(\sigma)$ the system can be switched from $q$ to $q^{\prime}$ by a discrete transition.

Depending on the characteristics of dynamics $\dot{x}_{q}=f_{q}(x, u, d)$ the following subclasses of hybrid automata can be defined:

- Linear Hybrid Automata (Xia et al., 2002): $\dot{x}_{q}(t)=u(t)+d(t)$ where $u(t) \in U_{q}$ and $d(t) \in D_{q}, U_{q}=\left\{u \mid C_{U_{q}} u \leq h_{U_{q}}, C_{U_{q}} \in\right.$ $\left.\mathbb{R}^{n_{u} \times n}, h_{U_{q}} \in \mathbb{R}^{n}\right\}$ and $D_{q}=\left\{d \mid C_{D_{q}} d \leq\right.$ $\left.h_{D_{q}}, C_{D_{q}} \in \mathbb{R}^{n_{d} \times n}, h_{D_{q}} \in \mathbb{R}^{n}\right\}$. The continuous dynamics have both control and disturbance input.

- Rectangular Hybrid Automata (Preußíg et al., 1998): $\dot{x}_{q}(t)=u$ where $u \in\left[\underline{U}_{q}, \bar{U}_{q}\right], \underline{U}_{q}, \bar{U}_{q} \in$ $\mathbb{R}^{n}$. The continuous dynamics are rectangular differential inclusions.

- Integrator Hybrid Automata (Xu and Antsaklis, 2003): $\dot{x}_{q}(t)=k_{q}, k_{q} \in \mathbb{R}^{n}$. The continuous variables evolve at a fixed speed.

- Time Automata(Alur et al., 2001): $\dot{x}_{q}(t)=1$. The continuous variables $x$ act as clocks.

For these types of hybrid automata, we assume that all the invariant sets, guard sets and target set are convex polyhedral sets. It is assumed that the continuous states are the same before and after a discrete transition i.e. $q\left(t^{-}\right) \neq q\left(t^{+}\right)$, with $x\left(t^{-}\right)=x\left(t^{+}\right)$. Also, we assume that all the discrete events are controllable.

We use $\xi$ to denote a hybrid trajectory. The set of hybrid trajectories starting from $(q, x)$ is denoted by $L(A,(q, x))$ while $L(A)$ denotes the set of all hybrid trajectories generated by the automaton $A$. In the following $\xi(t)$ is used to denote the hybrid state of the trajectory at a given time instant $t$ if no discrete transition happens at $t$. Otherwise, if a discrete transition happens at $t$, then $\xi\left(t^{-}\right)$and $\xi\left(t^{+}\right)$denotes the hybrid state before and after the transition respectively.

Definition 1. A hybrid controller is a map:

$$
\mathcal{C}: Q \times X \times T^{+} \rightarrow 2^{\Sigma \times U}
$$

where $T^{+}$denotes time. Here, we assume that the controller has a clock which can record time. The controller issues both discrete inputs $\mathcal{C}_{d}(q(t), x(t), t) \in 2^{\Sigma}$ and continuous inputs $\mathcal{C}_{c}(q(t), x(t), t) \in 2^{U}$.

\subsection{Problem statement}

The optimal control problem we considered in this paper is as follows: Given a initial state $\left(q_{0}, x_{0}\right)$ of a hybrid automaton $A$ and a cost function $J$ of a hybrid trajectory, find an optimal hybrid controller such that the controlled trajectory starting from $\left(q_{0}, x_{0}\right)$ reaches the target set $F$ with a minimal cost. The solvability of the above problem depends on the definition of the cost function and the characteristics of the given automaton $A$.

Given a trajectory $\left.\xi \in L\left(A,\left(q_{0}, x_{0}\right)\right)\right)$ and the target set $F \subset X \times Q$, the time that a trajectory $\xi$ starting from $\left(q_{0}, x_{0}\right)$ needs to reach $F$ is:

$T_{f}\left(\xi, F,\left(q_{0}, x_{0}\right)\right)= \begin{cases}t_{f} \quad \text { if } \quad \xi(0)=\left(q_{0}, x_{0}\right), \xi\left(t_{f}\right) \in F \wedge \\ \\ \left(\forall t \in\left[0, t_{f}\right), \xi(t) \in I N V \wedge \xi(t) \notin F\right) \\ +\infty \text { otherwise }\end{cases}$

Given a trajectory $\xi \in L\left(A,\left(q_{0}, x_{0}\right)\right)$, since we are only interested in the running cost incurred when the system evolves from $\left(q_{0}, x_{0}\right)$ to $F$, we compute the cost along $\xi$ during the time interval $\left[0, T_{f}\left(\xi, F,\left(q_{0}, x_{0}\right)\right)\right]$. Let $e_{j}(\xi)$ denote the $j$ th discrete transition and $n(\xi)$ denote the total number of discrete transitions of $\xi$ during the time interval $\left[0, T_{f}\left(\xi, F,\left(q_{0}, x_{0}\right)\right)\right]$. Then, the cost along $\xi$ is defined as:

$$
J(\xi)=\left\{\begin{array}{c}
\sum_{j=1}^{n(\xi)} J_{d}\left(e_{j}(\xi)\right)+\int_{0}^{t_{f}} J_{c}(q(t)) d t \quad \text { if } \\
+\infty \\
T_{f}\left(\xi, F,\left(q_{0}, x_{0}\right)\right) \text { is finite } \\
\text { otherwise }
\end{array}\right.
$$

The running cost introduced above involves the following two cost functions:

- $J_{d}: E \rightarrow \mathbb{Q}$ discrete transition cost function.

- $J_{c}: Q \rightarrow \mathbb{Q}$ continuous transition cost function.

Basically we assign costs to both discrete and continuous transitions. Note that there is no penalty on the control input and on the continuous state.

From definition (3), it is clear that if there is no trajectory from $\left(q_{0}, x_{0}\right)$ to $F$ without violating the invariant constraints, then the optimal cost $\min _{\xi \in L\left(A,\left(q_{0}, x_{0}\right)\right)} J(\xi)=+\infty$. In order to guarantee that the optimal cost is finite, a backward reachability analysis (Xia et al., 2002) is performed starting from the target set $F$. If the target is reachable from $\left(q_{0}, x_{0}\right)$, then the optimal cost is finite. Due to the fact that all discrete transitions are controllable, the procedure to find an optimal 
trajectory $\xi^{*}$ which minimizes $(3)$ is divided into the following steps :

(1) Find all discrete paths that connect $q_{0}$ with $q_{F}$, following the Dijkstra's algorithm (Martins et al., 1999) on the discrete graph.

(2) Find the optimal trajectory which minimizes $\int_{0}^{t_{f}} J_{c}(q(t)) d t$ for each discrete path.

(3) Among all optimal trajectories find the optimal hybrid trajectory $\xi^{*}$ which minimizes (3).

(4) Design the optimal hybrid controller $\mathcal{C}^{*}$.

Let $\Pi=\{\pi\}$ denote the set of all discrete paths from $q_{0}$ to $q_{F}$ :

$$
\begin{gathered}
\Pi=\left\{\pi \mid \exists \sigma, \exists N \in \mathbb{N}, j=0, \ldots, N-1, q_{N}=q_{F}\right. \\
\left.e_{j}=\left(q_{j}, \sigma, q_{j+1}\right) \in E \wedge \pi=\left\{q_{0}, \ldots, q_{N}\right\}\right\}
\end{gathered}
$$

Let $l(\pi)$ be the number of discrete transitions in a path $\pi \in \Pi$. It is clear that the sum of discrete transition costs is constant along any given path $\pi, J_{d}^{\pi}:=\sum_{j=1}^{l(\pi)} J_{d}\left(e_{j}^{\pi}\right)$. Then, the optimal solution of (3) can be represented as follows:

$$
\min _{\pi \in \Pi}\left(J_{d}^{\pi}+\min _{t_{j} \geq 0} \sum_{j=0}^{l(\pi)} J_{c}\left(q_{j}^{\pi}\right) t_{j}\right)
$$

where $t_{j}$ denotes the time spent in $q_{j}$. Let $J_{c}^{\pi}:=$ $\min _{t_{j} \geq 0} \sum_{j=0}^{l(\pi)} J_{c}\left(q_{j}^{\pi}\right) t_{j}$ be the optimal continuous cost along a path $\pi$. Under the assumption that $F$ is reachable from $\left(q_{0}, x_{0}\right)$, the optimal cost $J^{*}(\xi)$ of $L\left(A,\left(q_{0}, x_{0}\right)\right)$ can be found.

Algorithm 1.

$J_{\min }:=+\infty$
repeat $i=1,2, \ldots$
$\pi_{i}=$ ith - shortest - path $(A)$
if $\left(J_{\min }<J_{d}^{\pi_{i}}\right)$ or $\left(\pi_{i}=\emptyset\right)$ break
$\quad J^{\pi_{i}}=J_{d}^{\pi_{i}}+J_{c}^{\pi_{i}}$
$\quad$ if $\left(J^{\pi_{i}}<J_{\min }\right)$
$\quad J_{\min }=J^{\pi_{i}}$
end
end
return $J^{*}(\xi)=J_{\min }$

The algorithm uses the results from (Martins et al., 1999) to rank the $i$-th shortest discrete paths from the initial state to the target set in ascending order of the discrete cost, i.e. $J_{d}^{\pi_{i}} \leq J_{d}^{\pi_{i+1}}$ for $i=1,2, \ldots$. When $\pi_{i}=\emptyset$ then there are only $i-1$ paths in the graph of $A$.

Proposition 2. Under the assumption that the target set is reachable from $\left(q_{0}, x_{0}\right)$, algorithm 1 terminates within finite iterations.

Proof: If $\pi_{i}=\emptyset$ for $i \in \mathbb{N}$, this means that the graph of $A$ has a finite number of discrete paths, then it is obvious that algorithm 1 will terminate with finite iterations. On the other hand, as it is feasible to reach the target from $\left(q_{0}, x_{0}\right)$, then $J^{*}(\xi)$ is finite. Since $J_{\min }$ is monotonously decreasing and $J_{d}^{\pi_{i}}$ is monotonously increasing during each iteration, it can be induced that there exists an integer $i$ such that $J^{\pi_{i}}=J_{d}^{\pi_{i}}+J_{c}^{\pi_{i}}$ is optimal.

From algorithm 1, the attainability of the optimal solution for the optimal control problem depends on one's ability to derive $J_{c}^{\pi}$. It is a continuous cost minimization problem along the fixed path $\pi$. This problem is considered in the rest of the paper.

Remark 3. Notice that if $J_{c}\left(q_{j}^{\pi}\right)=1$ for all $j=0, \ldots, l(\pi)$ along the path $\pi$, the optimal control problem is transformed into a timeoptimal control problem as follows: $t_{\min }=J_{c}^{\pi}=$ $\min _{t_{j} \geq 0} \sum_{j=0}^{l(\pi)} t_{j}$, and $t_{m i n}$ is the minimal time that the hybrid automaton needs to reach the target set $\mathrm{F}$ starting from $\left(q_{0}, x_{0}\right)$ along the path $\pi$. Otherwise the problem can be considered as a weighted time-optimal control problem. The overall cost is a balance between discrete transitions cost and time cost.

Let $G_{q_{i-1}, q_{i}}^{\pi}=\left\{x \mid C_{i}^{\pi} x \leq h_{i}^{\pi}\right\}$ denote the transition guard from $q_{i-1}^{\pi}$ to $q_{i}^{\pi}$, with $i=1, \ldots, l(\pi)$, where $C_{i}^{\pi} \in \mathbb{R}^{n_{c} \times n}, h_{i}^{\pi} \in \mathbb{R}^{n_{c}}$. Also, let $X_{F}=$ $\left\{x \mid C_{F} x \leq h_{F}\right\}$ be the continuous part of the target set, with $C_{F} \in \mathbb{R}^{n_{f} \times n}, h_{F} \in \mathbb{R}^{n_{f}}$. For simplicity, we omit the symbol " $\pi$ ". Consider a discrete path $\pi$ with $l(\pi)$ transitions. Our objective is to compute the minimal cost $J_{c}^{\pi}$. The time-optimal problem for integrator hybrid automata has been studied in (Xu and Antsaklis, 2003). Next, the optimal control for rectangular hybrid automata and linear hybrid automata is considered.

\section{OPTIMAL SOLUTION FOR RECTANGULAR HYBRID AUTOMATA}

This section deals with the optimal control problem for rectangular hybrid automata. Before proceeding with the main theorem, we discuss the relationship between the open and closed loop control. In each discrete state $q$, there are two types of controllers that generate the control input, namely the open loop controllers and the closed loop (feedback) controllers. Let $u_{q} \in\left[\underline{U}_{q}, \bar{U}_{q}\right]$ denote the control input generated by an open loop controller and let $u_{q}(t) \in\left[\underline{U}_{q}, \bar{U}_{q}\right]$ denote the control input (time-variant) generated by a closed loop controller. The relationship between these two controllers follows the First Mean Value Theorem for Integrals, see (Pang and Spathopoulos, 2004).

It follows that along a path $\pi$, for any trajectory $\xi$ and a feedback control input $u(t)$, an alternative trajectory $\xi^{\prime}$ can always be found with open loop 
control input $u$, such that $J(\xi)=J\left(\xi^{\prime}\right)$ and $\xi\left(\sum_{i=0}^{j} t_{j}\right)=\xi^{\prime}\left(\sum_{i=0}^{j} t_{j}\right)$ for $j=0, \ldots, l(\pi)$ where $t_{j}$ is the time the system spends in each location of the path $\pi$. Therefore, both type of controllers yield the same optimal cost. The advantage in the above consideration is that each open loop control $u_{q i}$ can be treated as an existential $(\exists)$ quantifier. Thus, quantifier elimination can be employed in order to get a set of linear inequalities for the variable t.

The objective of the real quantifier elimination is to eliminate "unwanted" variables from an algebraic description. The "unwanted" variables may represent unknown real quantities. Quantifiers give expressive power but do not enlarge the class of sets defined by quantifier-free formulas. This implies that given a formula including quantifiers

$\varphi\left(x_{1}, \ldots x_{n}\right) \equiv \mathbf{Q}_{\mathbf{1}} u_{1}, \ldots \mathbf{Q}_{\mathbf{m}} u_{m} \psi\left(x_{1}, \ldots x_{n}, u_{1}, \ldots u_{m}\right)$

where $\mathbf{Q}_{\mathbf{i}} \in\{\exists, \forall\}$, there is always a logically equivalent quantifier free formula $\phi\left(x_{1}, \ldots, x_{n}\right)$ in the domain of the real numbers. A procedure computing such $\phi$ from $\varphi$ is called real quantifier elimination.

Given a rectangular hybrid automaton $A$, let the initial state be $x_{0}=\left(x_{01}, \ldots, x_{0 n}\right)$, the dynamics of a discrete state $q_{j} \in \pi$ be $\dot{x}_{j}=u_{j}=$ $\left[u_{j 1}, u_{j 2}, \ldots, u_{j n}\right]^{T}$ for all $j=0, \ldots, l(\pi)$, with control input $u_{j} \in\left[\underline{U}_{j}, \bar{U}_{j}\right], \underline{U}_{j}, \bar{U}_{j} \in \mathbb{R}^{n}$.

Theorem 4. The optimal continuous cost along a path $\pi$ for a rectangular automaton $A$ with parameters defined above, can be computed by

$$
\begin{aligned}
& J_{c}^{\pi}=\min \lambda^{T} t \\
& \text { s.t. } \phi(t)
\end{aligned}
$$

The quantifier free formula $\phi(t)$ that is linear in $t$ is computed from $\varphi(t)$ by quantifier elimination in real domain where:

$$
\begin{array}{r}
\varphi(t) \equiv \exists u_{11}, \ldots, \exists u_{1 n}, \exists u_{21}, \ldots, \exists u_{2 n}, \ldots, \exists u_{l(\pi) 1}, \ldots, \\
\\
\exists u_{l(\pi) n}\left(\psi_{1}(u, t) \wedge \psi_{2}(u, t) \wedge \psi_{3}(t) \wedge \psi_{4}(u)\right)
\end{array}
$$

and:

$$
\begin{aligned}
\psi_{1}(u, t) & =\bigwedge_{i=1}^{l(\pi)} C_{i}\left(\left[x_{01}, \ldots, x_{0 n}\right]^{T}+\right. \\
& \left.\sum_{j=0}^{i}\left[u_{j 1}, \ldots, u_{j n}\right]^{T} t_{j}\right) \leq h_{i}(\text { guard condition }) \\
\psi_{2}(u, t)= & C_{F}\left(\left[x_{01}, \ldots, x_{0 n}\right]^{T}+\right. \\
& \left.\sum_{j=0}^{l(\pi)}\left[u_{j 1}, \ldots, u_{j n}\right]^{T} t_{j}\right) \leq h_{F}(\text { target condition }) \\
\psi_{3}(t)= & \bigwedge_{j=1}^{l(\pi)} t_{j} \geq 0(\text { nonnegative time condition }) \\
\psi_{4}(u)= & \bigwedge_{j=1}^{l(\pi)} \bigwedge_{r=1}^{n} \underline{U}_{j r} \leq u_{j r} \leq \bar{U}_{j r}(\text { input restrictions })
\end{aligned}
$$

Proof: See (Pang and Spathopoulos, 2004).

It should be noted that since the two variables $t$ and $u$ are nonlinear in the formulas $\psi_{1} \sim \psi_{4}$, it is impossible to solve the LP problem. Nevertheless, by considering the continuous input $u$ as existential $(\exists)$ quantifier, we get an equivalent formula $\phi$ by eliminating the $u$ quantifier. According to the quantifier elimination procedure, the quantifier free formula $\phi$ is formulated as linear inequalities whose closure is a convex set.

As mentioned early on, in order to guarantee the optimal performance, an optimal controller has to be imposed on the system. As the LP (5) converges to an optimal solution, we get the optimal control switching times $t^{*}$. Then the continuous optimal control law $U^{*}$ can be obtained using the following two-step procedure:

- Step1: substitute the optimal time $t^{*}$ derived from LP into the formulas $\psi_{1}, \psi_{2}, \psi_{4}$.

- Step2: simplify these formulas to eliminate redundant inequalities.

With the optimal times $t_{0}^{*}, \ldots, t_{l(\pi)}^{*}$ obtained from the LP problem 4, the optimal controller is:

$$
\mathcal{C}^{*}(q(t), x(t), t)=\left\{\begin{aligned}
\left(\epsilon, u_{0}^{*}\right) \quad & \text { if } 0 \leq t<t_{0}^{*} \\
& \wedge q(t)=q_{0} \wedge u_{0}^{*} \in U_{0}^{*} \\
\left(\sigma_{j, j+1}, \emptyset\right) & \text { if } t=\sum_{i=0} t_{i}^{*} \wedge q\left(t^{-}\right)=q_{j} \\
& \wedge\left(q_{j}, \sigma_{j, j+1}, q_{j+1}\right) \in E \\
& \text { for } j=0, . ., l(\pi)-1 \\
& \text { if } \sum_{i=0}^{j-1} t_{i}^{*} \leq t<\sum_{i=0}^{j} t_{i}^{*} \\
\left(\epsilon, u_{j}^{*}\right) \quad & \wedge q(t)=q_{j} \wedge u_{j}^{*} \in U_{j}^{*} \\
& \text { for } j=1, . ., l(\pi) \\
&
\end{aligned}\right.
$$

It should be noted that the set $U_{j}^{*}$, obtained from above two-step procedure, is the optimal continuous input set for the discrete state $q_{j}^{\pi}$. The clock is set to 0 at the initial state $\left(q_{0}, x_{0}\right)$. During the time $t \in\left[\sum_{i=0}^{j-1} t_{i}^{*}, \sum_{i=0}^{j} t_{i}^{*}\right)$ there is no discrete input and the continuous input is $u_{j}^{*} \in U_{j}^{*}$. At the optimal time $t=\sum_{i=0}^{j} t_{i}^{*}$ the discrete optimal controller generates a suitable discrete event to switch the system to the next discrete state $q_{j+1}$ along the path $\pi$. At the same time the continuous optimal controller chooses a continuous input from $U_{j+1}^{*}$ as soon as the system has been switched to the discrete state $q_{j+1}$.

\section{OPTIMAL SOLUTION FOR LINEAR HYBRID AUTOMATA}

If we consider linear hybrid automata with both control and disturbance inputs the problem becomes more difficult. The optimal control problem can not be solved using quantifier elimination when a feedback control is required. However, we 
can still get an open loop controller in the sense that the control input $u$ is fixed at each discrete state $q$.

Due to the presence of disturbances, the open loop control is optimal in the worst case sense. The dynamics in a discrete state $q_{j} \in \pi$ become $\dot{x}_{j}(t)=u_{j}+d_{j}(t)$ for all $j=0, \ldots, l(\pi)$, with control input $u_{j} \in U_{j}, U_{j}=\left\{u \mid C_{U_{j}} u \leq h_{U_{j}}, C_{U_{j}} \in\right.$ $\left.\mathbb{R}^{n_{u} \times n}, h_{U_{j}} \in \mathbb{R}^{n}\right\}$ and disturbance $d_{j}(t) \in D_{j}$, $D_{j}=\left\{d \mid C_{D_{j}} d \leq h_{D_{j}}, C_{D_{j}} \in \mathbb{R}^{n_{d} \times n}, h_{D_{j}} \in \mathbb{R}^{n}\right\}$. Let $d_{j m}\left(m=1,2, \ldots, p_{j}\right)$ denote the vertices of the set $D_{j}$

Theorem 5. The worst case optimal continuous cost along a path $\pi$ from an initial state $x_{0}$ for linear hybrid automata with both control and disturbance input can be computed as

$$
\begin{aligned}
& J_{c}^{\pi}=\min \lambda^{T} t \\
& \text { s.t. } \phi(t)
\end{aligned}
$$

The quantifier free formula $\phi(t)$ is computed from $\varphi(t)$ by quantifier elimination in real domain, where

$$
\begin{aligned}
& \varphi(t) \equiv \exists u_{11}, \ldots, \exists u_{1 n}, \exists u_{21}, \ldots, \exists u_{2 n}, \ldots, \exists u_{l(\pi) 1}, \\
& . ., \exists u_{l(\pi) n}\left(\psi_{1}(u, t) \wedge \psi_{2}(u, t) \wedge \psi_{3}(t) \wedge \psi_{4}(u)\right)
\end{aligned}
$$

and:

$$
\psi_{1}(u, t)=\bigwedge_{i=1}^{l(\pi)} \overbrace{\bigwedge_{m=1}^{p_{j}} \ldots \bigwedge_{m=1}^{j=0, . ., i}}^{\bigwedge_{p_{j}}}\left(C_{i}\left(x_{0}+\sum_{j=0}^{i}\left(u_{j}+d_{j m}\right) t_{j}\right) \leq h_{i}\right.
$$$$
\psi_{2}(u, t)=\underbrace{\bigwedge_{m=1}^{p_{j}} \ldots \bigwedge_{m=1}^{p_{j}}}_{j=0, . ., l(\pi)} C_{F}\left(x_{0}+\sum_{j=0}^{l(\pi)}\left(u_{j}+d_{j m}\right) t_{j}\right) \leq h_{F}
$$$$
\psi_{3}(t)=\bigwedge_{j=1}^{l(\pi)} t_{j} \geq 0
$$$$
\psi_{4}(u)=\bigwedge_{j=1}^{l(\pi)} C_{U_{j}} u_{j} \leq h_{U_{j}}
$$

Proof: See (Pang and Spathopoulos, 2004).

The optimal continuous control law $U^{*}$ for linear hybrid automata can also be designed following the two-step procedure presented above. The optimal controller $\mathcal{C}^{*}$ is the same with the one derived for rectangular hybrid automata as in (6).

\section{APPLICATION OF AIR TRAFFIC MANAGEMENT}

In this section, the results of optimal control are applied on an Air Traffic Management System (ATMS) (Tomlin, 1996), (Pang and Spathopoulos, 2004). This two aircraft joining and collision

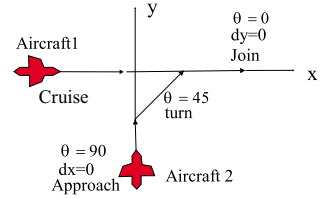

Fig. 1. One aircraft joining the path of another, in an approach to the airport runway

avoidance scheme can be seen as an optimal control problem. The scenario is a common one and illustrated in figure 5 . Aircraft 2 is joining the path of Aircraft 1, in approach to the airport, and it is up to the controller to ensure that a minimal separation distance between the two aircraft is maintained and also minimize the cost during this procedure. Aircraft 1 has one mode of operation, called Cruise: it follows a straight path along the $\mathrm{x}$-axis and has known bounds on its speed. Aircraft 2 is in three possible position modes: Approach, Turn and Join. Within the Turn and Join modes, Aircraft 2 can be in one of three possible speed modes, the controller effects both the transitions and the speed of the modes. The control scheme is as follows: if the $\mathrm{x}$ coordinate of Aircraft 2 is either greater than that of Aircraft 1 or more than 30 units behind that of Aircraft 1 , then the Aircraft 2 should be in a fast mode. If the $\mathrm{x}$ coordinate of Aircraft 2 is less than 30 units behind that of Aircraft 1, then the Aircraft 2 should be in a slow mode. There are two fast modes and one slow mode: Fast 1 is the mode in which Aircraft 2 is more than 30 units behind Aircraft 1. Fast2 is the mode in which Aircraft 2 is ahead of Aircraft 1.

The speed of Aircraft 1 is $\dot{x}=u_{1}$, where $u_{1} \in[3,4]$ in any position mode. For simplicity, we restrict the transitions between the Fast and slow modes if they are in the same position mode. Then the aim of this air traffic management is that Aircraft 2 join the path of Aircraft 1 safely with $x 1 \geq 50 \wedge x 2 \geq 50$. The safety requirement is that the distance between the two aircraft has to be kept over 10. That is roughly illustrated by the forbidden region $B a d=\{|x 1-x 2| \leq 10 \wedge$ $y 2 \leq 10\}$.

It should be noted that the problem requires the composing the motions of both Aircraft 1 and Aircraft 2. The composed automaton has three discrete position modes of Aircraft 2. Its continuous states are in 3 dimensions: $(x 1, x 2, y 2)$. We assume that the two aircraft communicate very well, and the Aircraft 1 can also change its speed when Aircraft 2 changes its position modes. Hence, by composing the two systems we get the target set $\mathrm{F}=($ Join, $F 1 \cup F 2)$ for the composed hybrid system where the discrete state of Aircraft 2 is Join and the continuous sets are either $F 1=$ $\{x 1-x 2 \geq 10 \wedge x 2 \geq 50\}$ or $F 2=\{x 2-x 1 \geq 10 \wedge$ $x 1 \geq 50\}$ 


\begin{tabular}{|c|c|c|}
\hline Path & $\pi_{1}$ & $\pi 2$ \\
\hline$q_{0}^{\pi}$ & $\begin{array}{c}A p p f \\
x 2=0 \\
-100 \leq y 2 \leq-30 \\
\dot{x} 2=0 \\
\exists \dot{y} 2 \in[6,7] \\
\exists \dot{x} 1 \in[3,4]\end{array}$ & $\begin{array}{c}\text { Appf } \\
x 2=0 \\
-100 \leq y 2 \leq-30 \\
\dot{x} 2=0 \\
\exists \dot{y} 2 \in[6,7] \\
\exists \dot{x} 1 \in[3,4]\end{array}$ \\
\hline$q_{1}^{\pi}$ & $\begin{array}{c}\text { Turns } \\
0 \leq x 2 \leq 30 \\
-30 \leq y 2 \leq 0 \\
x 2 \leq x 1 \leq 30+x 2 \\
\exists \dot{x} 2 \in[4,5] \\
\dot{y} 2=\dot{x} 2 \\
\exists \dot{x} 1 \in[3,4]\end{array}$ & $\begin{array}{c}\text { Turnf } 2 \\
0 \leq x 2 \leq 30 \\
-30 \leq y 2 \leq 0 \\
x 2 \geq x 1 \\
\exists \dot{x} 2 \in[5,6] \\
\dot{y} 2=\dot{x} 2 \\
\exists \dot{x} 1 \in[3,4]\end{array}$ \\
\hline$q_{2}^{\pi}$ & $\begin{array}{c}\text { Joins } \\
x 2 \geq 30 \\
y 2=0 \\
x 2 \leq x 1 \leq 30+x 2 \\
\exists \dot{x} 2 \in[4,5] \\
\dot{y} 2=0 \\
\exists \dot{x} 1 \in[3.5,4]\end{array}$ & $\begin{array}{c}\text { Joinf } 2 \\
x 2 \geq 30 \\
y 2=0 \\
x 2 \geq x 1 \\
\exists \dot{x} 2 \in[5,6] \\
\dot{y} 2=0 \\
\exists \dot{x} 1 \in[3,4]\end{array}$ \\
\hline$G_{q_{0}^{\pi} q_{1}^{\pi}}$ & $\begin{array}{c}x 2 \leq x 1 \leq 30+x 2 \\
y 2=-30\end{array}$ & $\begin{array}{c}x 2 \geq x 1 \\
y 2=-30\end{array}$ \\
\hline$G_{q_{1}^{\pi} q_{2}^{\pi}}$ & $\begin{array}{c}x 2=30 \\
y 2=0 \\
10 \leq x 1-x 2 \leq 30\end{array}$ & $\begin{array}{c}x 2=30 \\
y 2=0 \\
x 2-x 1 \geq 10\end{array}$ \\
\hline Target & $\overline{(J o i n s, F 1)}$ & $(J o i n f 2, F 2)$ \\
\hline$J_{d}^{\pi}$ & $20+20=40$ & $20+20=40$ \\
\hline $\begin{array}{l}\text { Optimal } \\
\text { time } t^{*}\end{array}$ & $\begin{array}{c}t_{0}^{*}=11.5457 ; t_{1}^{*}=6 \\
t_{2}^{*}=4.9543\end{array}$ & $\begin{array}{c}t_{0}^{*}=10 ; t_{1}^{*}=5 \\
t_{2}^{*}=7.5\end{array}$ \\
\hline$J_{c}^{\pi}$ & 51 & 50 \\
\hline$J^{\pi}$ & 91 & 90 \\
\hline
\end{tabular}

Table 1. Final results of two paths.

\begin{tabular}{|c|c|}
\hline Mode & Controller \\
\hline Appf & $\dot{x} 1=u_{10}^{*}=3, \dot{y} 2=u_{20}^{*}=7$ \\
\hline Turnf2 & $\dot{x} 1=u_{11}^{*}=4, \dot{x} 2=\dot{y} 2=u_{21}^{*}=6$ \\
\hline Joinf2 & $\dot{x} 1=u_{12}^{*}=4, \dot{x} 2=u_{22}^{*} \in[5,6]$ \\
\hline
\end{tabular}

The optimal control for this air traffic management is cast as follows: the two aircraft reach the target set $\mathrm{F}$ without entering the forbidden region Bad at minimal cost. Here, we associate each transition with the discrete cost $J_{d}=20$. The continuous costs in Approach and Join position modes are $J_{c}($ Approach $)=J_{c}($ Join $)=2$, and the continuous cost in the Turn position mode is $J_{c}($ Turn $)=3$. Given an initial state in Approach mode: $x 1(0)=-30, x 2(0)=0, y 2(0)=-100$, there are only two discrete paths acceptable (the others generate blocking or lead the system to the forbidden region), namely $\pi_{1}=\{$ Appf Turns Joins $\} \pi 2=\{$ Appf Turnf 2 Joinf 2$\}$. The details of these paths are shown in table 1 . It follows that the optimal cost is $J^{\pi_{2}}=90$ with the optimal schedule $t^{*}$ as shown in table 1 . The optimal continuous inputs of Aircraft 1 and Aircraft 2 along the path $\pi_{2}$ are illustrated in table 2 and the optimal trajectories $\xi^{*}$ in $3 \mathrm{D}$ are shown in figure 2 .

\section{CONCLUSIONS}

We show how quantifier elimination can be applied in order to derive the appropriate optimal control strategy in the presence of continuous disturbances. A two aircraft joining and collision avoidance system is designed optimally using our method. The proposed linear hybrid automata

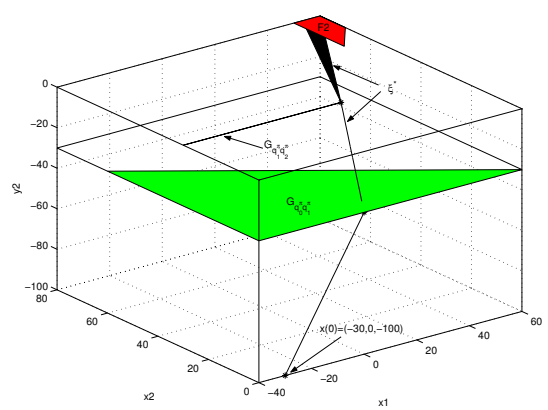

Fig. 2. Optimal trajectories in 3D.

have relative simple dynamics. Optimal control for the decidable classes of linear hybrid systems stated in (Lafferrire et al., 2001) should be further investigated.

\section{REFERENCES}

Alur, R., S. La Torre and G.J. Pappas (2001). Optimal paths in weighted timed automata. In: Hybrid Systems: Computation and Control. LNCS 2034. Springer-Verlag.

Collins, G. and H. Hong (1991). Partial cylindrical algebriac decomposition for quantifier elimanation. Journal of Symbolic Computation 12, 299-328.

Lafferrire, G., G. Pappas and S. Yovine (2001). Symbolic reachability computation for families of linear vector fields. Journal of Symbolic Computation 32, 231-253.

Martins, E., M. Pascoal and J. Dos Santos (1999). Labelling algorithms for ranking shortest paths. Technical report. Department of Mathematics, Coimbra University. Portugal.

Pang, Y. and M. . Spathopoulos (2004). Reachability and optimal control for linear hybrid automata: A quantifier elimination approach. Technical report. University of Strathclyde (Submitted).

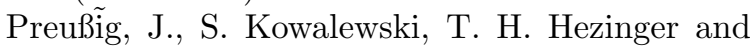
H. Wong-Toi (1998). An algorithm for the approximative analysis of simple rectangular automata. In: Formal Techniques for Real-Time and Falt-Tolerant Systems. LNCS. Spring-Verlag.

Tomlin, C. (1996). Verification of air traffic management protocol using hytech. Technical report. EE290A, Henzinger- Department of Electrical Engineering and Computer Science, University of California at Berkelay.

Xia, H., Y. Pang, A. Trontis and M. Spathopoulos (2002). Eventuality synthesis for controlled linear automata. In: Proceedings of the American Control Conference. Anchorage, Alaska.

Xu, X. and P.J. Antsaklis (2003). A linear programming approach to time optimal control of integrator switched systems with state constraints. In: Proceedings of the 42nd Conference on Decision and Control. Hawaii, USA. 